\title{
Fabrication of functional microstructured optical fibers through a selective-filling technique
}

\author{
Yanyi Huang, Yong $\mathrm{Xu},{ }^{\text {a) }}$ and Amnon Yariv \\ Department of Applied Physics, California Institute of Technology, Mail Code 128-95, Pasadena, California \\ 91125
}

(Received 6 May 2004; accepted 4 October 2004)

\begin{abstract}
We develop a method for the fabrication of functional microstructured optical fibers (MOFs) by selectively filling the air holes with liquid phase materials, where we utilize the dependence of filling speed on the size of the air holes. As a demonstration, we construct a hybrid MOF by filling the center hollow core of a triangular lattice photonic crystal fiber with dye-doped curable polymer, and experimentally observe the two-photon fluorescence from the hybrid MOF. () 2004 American Institute of Physics. [DOI: 10.1063/1.1828593]
\end{abstract}

Confinement of light in low index core (air for example) using Bragg reflection was proposed in 1978. ${ }^{1}$ More recently, microstructured optical fibers (MOFs) inspired by photonic band-gap research have been extensively investigated, leading to many interesting optical phenomena. ${ }^{2-4} \mathrm{~A}$ hollow-core MOF can achieve air guiding by using the Bragg reflection from the periodic fiber cladding structure, ${ }^{1}$ which can potentially lead to ultralow propagation $\operatorname{loss}^{5}$ and have a significant impact on long-distance optical communications. In addition, hollow-core MOFs are an attractive medium for the study of nonlinear optical phenomena in gas phase materials. ${ }^{6}$ Solid-core MOFs, which are another class of MOFs where optical confinement is provided by effective index guiding, have also found a wide range of applications such as dispersion compensation and supercontinuum generation. ${ }^{7}$ Since both classes of MOFs are typically composed of an array of air holes in a solid material (such as silica), they also provide an excellent platform for the infiltration of liquid phase materials and the construction of microfluidic photonic devices such as tunable optical filters ${ }^{8,9}$ and switches. ${ }^{10}$ However, the microfluidic photonic devices reported to date use silica-core MOFs as templates, where all the surrounding air holes in the MOF are filled with liquid phase materials and light is guided within the center silica core. In this letter we report on unique method which allows us to selectively fill the air holes in air-core MOFs and form various functional hybrid MOFs for different applications. More specifically, we demonstrate a hybrid MOF in which only the central hollow core is filled with a functional material while the cladding structure is mostly air. Such a hybrid MOF, which can be well approximated by an optical fiber with the functional material as the fiber core and a mostly air cladding structure, is fundamentally different from other microfluidic MOFs reported in the literature as discussed later.

The process of fabricating a hybrid MOF begins with a template hollow-core MOF, in which the size of the central core differs significantly from that of the air holes in the MOF cladding. In our example, the MOF template is a hollow-core triangular lattice photonic crystal fiber, as illustrated in Fig. 1(a). The fiber is fabricated by Crystal Fibre A/S (Denmark) using a stack and draw technique. The scanning electron microscope (SEM) images of the fiber cross

\footnotetext{
${ }^{a)}$ Electronic mail: yong@caltech.edu
}

section are shown in Figs. 1(b) and 1(c). The diameters of the central hole and cladding holes are 5.8 and $2.8 \mu \mathrm{m}$, respectively. From the SEM images, we estimate that the thickness of the silica layers that separate adjacent cladding air holes is of the order of $50-100 \mathrm{~nm}$. The air filling fraction of the fiber cladding structure is approximately $90 \%$. This MOF template is originally designed for supporting hollow-core guiding within the wavelength range of $1.1-1.3 \mu \mathrm{m}$.

To achieve selective air-hole filling for the MOF template shown in Fig. 1, we employ a UV curable polymer and a multistep injection-cure-cleave process. A schematic flowchart of this process is illustrated in Fig. 2(a). In the first step, we inject Norland optical adhesive 73 (NOA73, viscosity $130 \mathrm{cps}$ at room temperature, UV curable) into the holes of the MOF (including the core) with a syringe. The force that drives the polymer into the fiber air holes is provided by the application of an external pressure on the syringe pump. Due to the high viscosity of the NOA73, the capillary force is insignificant in the process of polymer infiltration. We can model the liquid injection process as laminar flow in the cylindrical holes, which leads to ${ }^{11}$

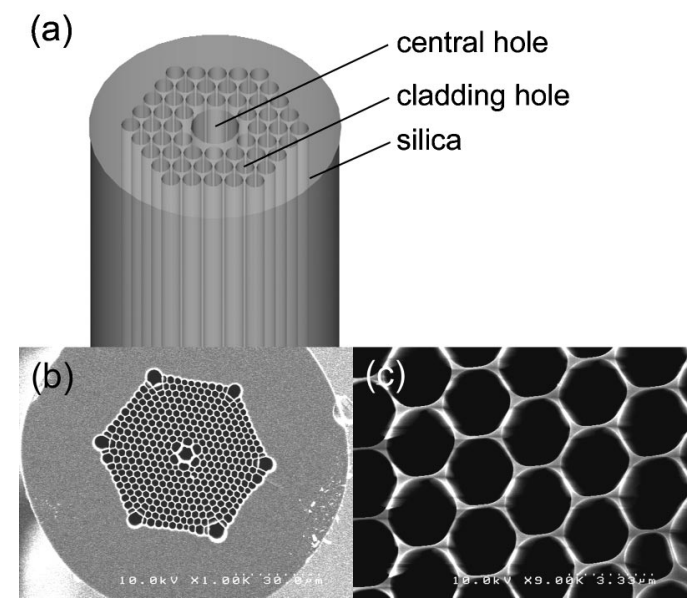

FIG. 1. Structure of the microstructured optical fiber. Two differently sized holes are shown in the schematic diagram (a). The SEM images of the fiber cross section show both the central hole and cladding holes (b), as well as the triangular lattice of the cladding holes (c). 

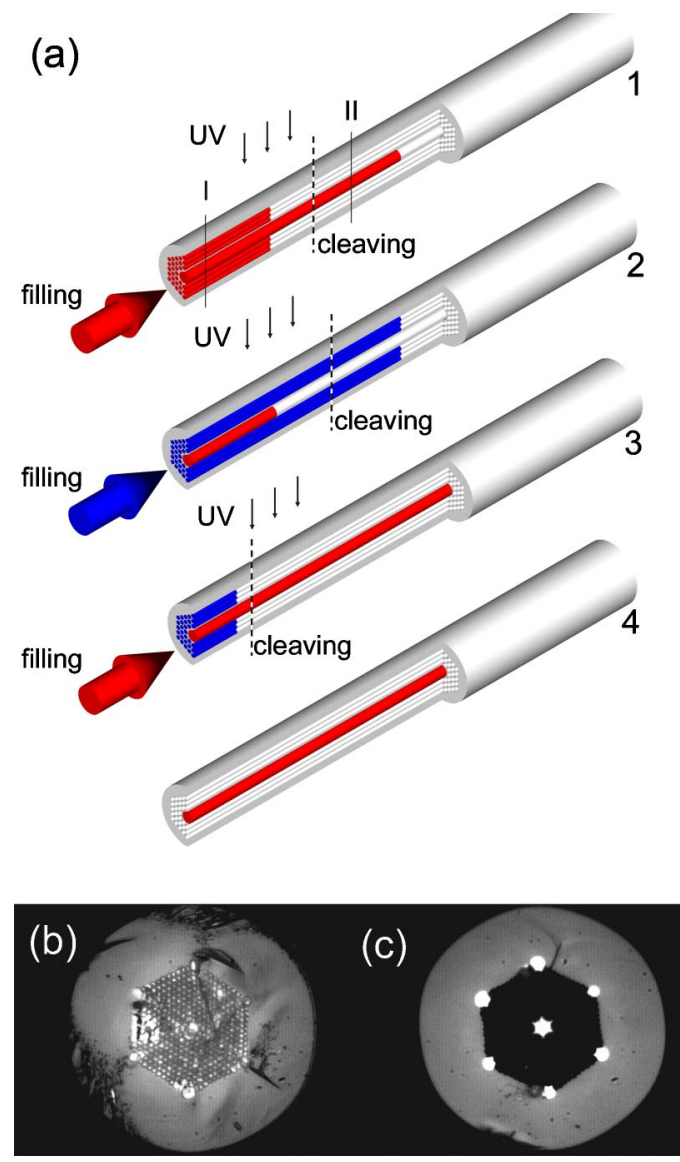

FIG. 2. (Color online) Schematic flowchart on selective filling of the microstructured optical fibers. The images (b) and (c) are optical microscope images of the fiber cross sections at the cleave positions I and II. The light regions correspond to the holes filled with polymer.

$$
v=-\frac{\Delta p r^{2}}{8 l \mu},
$$

where $v$ is the average velocity of the liquid flow in the air hole, $r$ is the radius of the hole, $\Delta p$ is the pressure difference between the two ends of the liquid tube within the cylindrical hole, $l$ is the infiltration length of the liquid within the hole, and $\mu$ is the viscosity of the liquid. Equation (1) indicates that the liquid fills the larger holes much faster than the smaller holes. After a given period of time, the infiltration length of NOA73 within the central hole is longer than that within the cladding holes (step 1). Consequently, along the certain length from the input of the MOF template [position I in step 1, Fig. 2(a)], all the holes are filled with NOA73 as shown in Fig. 2(b). However, beyond that portion [position II in step 1, Fig. 2(a)], only the bigger holes are filled with NOA73, as shown in Fig. 2(c). We also notice that due to the imperfect fabrication, in Fig. 2(c) there are six holes around the edge of the cladding structure with a size comparable to that of the central hole. However, filling the six holes at the edge does not significantly affect the optical properties of the final hybrid MOF. We subsequently UV cure the NOA73 inside the MOF and cleave the fiber at the position shown in step 1, which results in a modified MOF with the central hole capped by the cured polymer. According to SEM images, there is no noticeable delamination between the polymer and the inner walls of the air holes after the curing, which indicates that the volume shrinkage of NOA73 during the UV curing is negligible. With the presence of the cap, in step 2 Downloaded 14 Dec 2005 to 131.215.225.171. Redistribution subject

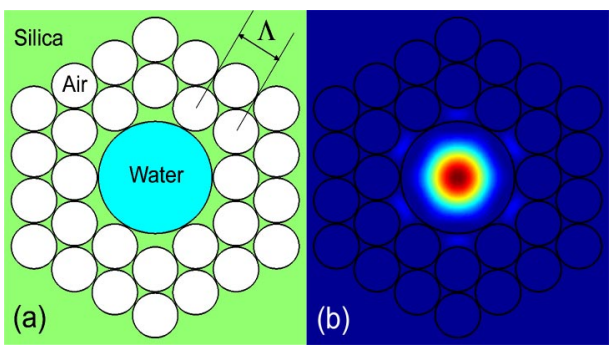

FIG. 3. (Color online) The structure (a) and the profile of a guided mode (b) in a selectively filled silica microstructured optical fiber $(\Lambda=3.1 \mu \mathrm{m})$. The central hole (radius $3.78 \mu \mathrm{m}$ ) is filled with water while the cladding holes (radius $1.51 \mu \mathrm{m}$ ) are filled with air.

we can selectively inject any liquid or solution into the MOF cladding holes (in our specific example, we again choose NOA73). We apply pressure on the syringe pump so the liquid injected in the cladding holes penetrates beyond the length of the cap in the central hole. We then cure the NOA73 in the cladding holes and cleave the fiber at the position shown in step 2, Fig. 2(a). At the end of the second step, we have capped all cladding holes with cured polymer but left the central hole open. Finally, in step 3, we can inject liquid selectively into the central hole with the cladding holes plugged. After cleaving [as shown in Fig. 2(a), step 3], we obtain a hybrid MOF with the central hole filled with the desired functional material while the fiber cladding consists of an array of air holes in silica.

Compared with other types of MOFs, the hybrid MOF described above has several distinct advantages. First, compared with silica-based MOFs, the hybrid MOF core can be made from various materials with nonlinear optical coefficients several orders of magnitude higher than those of silica glass, which makes the hybrid MOF very attractive for nonlinear optics applications. Furthermore, for a hybrid MOF with a cladding air filling fraction close to 1 , we can achieve optical guiding in low index materials such as water. In Fig. 3 , we show the profile of a guided mode in a hybrid MOF in which the central core is filled with water, and the cladding is composed of a triangular lattice of air holes in silica. The mode profile is calculated using the multiple expansion method. ${ }^{12}$ Since the optical field is well confined within the low index (water) core, we can achieve a long effective interaction length between the optical field and the low index material, which is important for spectroscopy applications in water-based solutions. The only other optical fiber that can guide light in a water-based solution core is the Teflon AFcoated capillary tube. ${ }^{13}$ However, compared with the Tefloncoated fiber, the hybrid MOF fiber possesses some important advantages in terms of the ease of fabrication, a much larger numerical aperture (therefore higher collection efficiency of luminescence), and a much smaller core radius, which leads to higher optical intensity and is important for nonlinear spectroscopy.

We demonstrate an example of functional MOFs using the selective-filling technique described above. The optical microscope image of the MOF cross section is shown in Fig. 2(c). In this demonstration, the core material is UV cured NOA73 doped with 0.05 wt \% (0.0002 mol/L) Rhodamine 640 (R640). It should be mentioned that light in this MOF is confined by total internal reflection instead of Bragg reflection. Compared with the alternative approach, where a glass capillary tube is used, the selectively filled MOF shown in to AIP license or copyright, see http://apl.aip.org/apl/copyright.jsp 

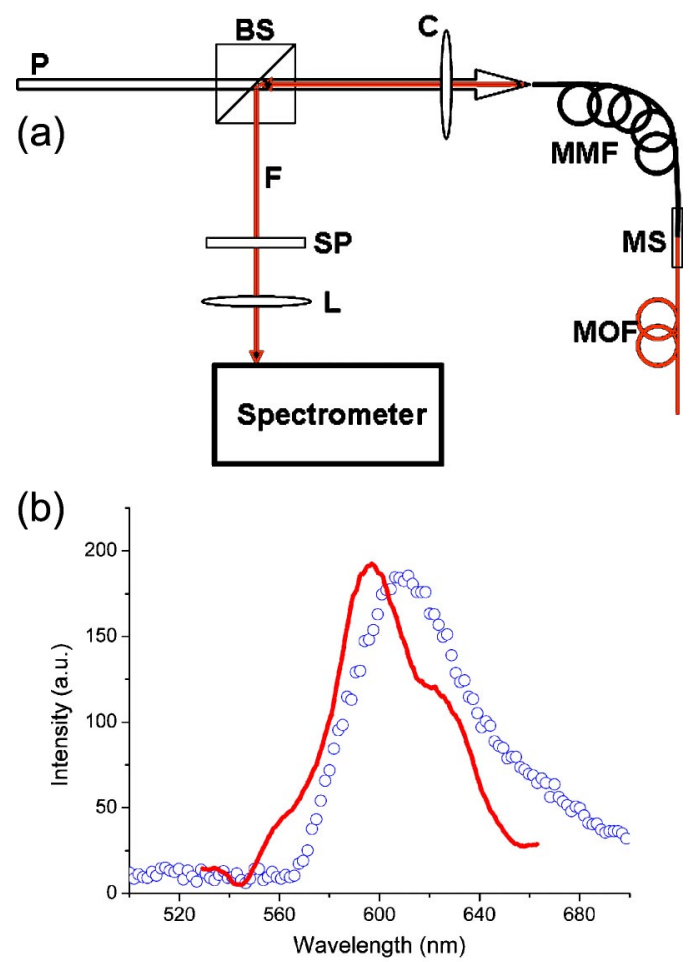

FIG. 4. (Color online) Experimental setup for optical measurement (a), $P$, pump; BS, beam splitter; $C$, collimator; MMF, multimode fiber; MS, mechanical splicer; $F$, fluorescence; SP, short-pass filter; $L$, lens. Detected fluorescence spectra (b) correspond to single-photon (532 nm, open circle) and two-photon (890 nm, solid line) pumping, respectively.

Fig. 2(c) can achieve much better optical confinement. Furthermore, the MOF can also realize optical confinement in polymer or liquid material with low $(n<1.44)$ or very low $(n<1.33)$ refractive index. The MOF can be handled in the same way as common telecommunication fibers. To measure the two-photon fluorescence from the MOF, we use the optical setup shown in Fig. 4. A 890 -nm fs laser (200 fs, 76 $\mathrm{MHz}, 15 \mathrm{~mW}$ ) is used as the pump. Since the cladding of the template MOF consists mostly of air [Fig. 1(c)], the pump light is well confined within the polymer core due to index guiding, and excites the entire length of the hybrid MOF (total length $20 \mathrm{~cm}$ ). The two-photon excited fluorescence that travels backward is collected and detected as shown in Fig. 4(a). The fluorescence spectrum of the hybrid MOF is shown in Fig. 4(b). Compared with conventional methods, ${ }^{14,15}$ our demonstration provides a unique method for fabricating highly efficient two-photon fluorescence fiber devices.

In summary, we propose and develop a method that allows the selective filling of MOFs with various functional materials. In particular, the selective-filling technique allows for the construction of a hybrid MOF in which the cladding structure is nearly all air and the central light-guiding core can be made from almost any solution-based materials. Using such a hybrid MOF, we can achieve optical confinement in low index materials such as water, which is of particular importance for spectroscopy and sensing applications in water-based solutions.

The authors wish to thank Professor S. R. Quake, Dr. R. K. Lee, J. K. S. Poon, J. M. Choi, W. M. J. Green, W. Liang, and G. T. Paloczi for fruitful discussions and kind help. This work is supported by the National Science Foundation and Defence Advanced Research Projects Agency (Dr. D. Honey and Dr. R. Athale).

${ }^{1}$ P. Yeh, A. Yariv, and E. Marom, J. Opt. Soc. Am. 68, 1196 (1978).

${ }^{2}$ P. Russell, Science 299, 358 (2003).

${ }^{3}$ J. C. Knight, Nature (London) 424, 847 (2003).

${ }^{4}$ J. Zagari, A. Argyros, N. A. Issa, G. Barton, G. Henry, M. C. J. Large, L. Poladian, and M. A. van Eijkelenborg, Opt. Lett. 29, 818 (2004).

${ }^{5}$ C. M. Smith, N. Venkataraman, M. T. Gallagher, D. Muller, J. A. West, N. F. Borrelli, D. C. Allan, and K. W. Koch, Nature (London) 424, 657 (2003).

${ }^{6}$ F. Benabid, J. C. Knight, G. Antonopoulos, and P. S. J. Russell, Science 298, 399 (2002)

${ }^{7}$ J. Herrmann, U. Griebner, N. Zhavoronkov, A. Husakou, D. Nickel, J. C. Knight, W. J. Wadsworth, P. S. J. Russell, and G. Korn, Phys. Rev. Lett. 88, 173901 (2002).

${ }^{8}$ C. Kerbage and B. J. Eggleton, Appl. Phys. Lett. 82, 1338 (2003).

${ }^{9}$ P. Domachuk, H. C. Nguyen, B. J. Eggleton, M. Straub, and M. Gu, Appl. Phys. Lett. 84, 1838 (2004).

${ }^{10}$ T. T. Larsen, A. Bjarklev, D. P. Hermann, and J. Broeng, Opt. Express 11, 2589 (2003).

${ }^{11}$ R. H. Sabersky, A. J. Acosta, E. G. Hauptmann, and E. M. Gates, Fluid Flow, 4th ed. (Prentice Hall, Upper Saddle River, NJ, 1999).

${ }^{12}$ T. P. White, B. T. Kuhlmey, R. C. McPhedran, D. Maystre, G. Renversez, C. M. de Sterke, and L. C. Botten, J. Opt. Soc. Am. B 19, 2322 (2002).

${ }^{13}$ Z. Liu and J. Pawliszyn, Anal. Chem. 75, 4887 (2003).

${ }^{14}$ G. S. He, J. D. Bhawalkar, C. F. Zhao, C. K. Park, and P. N. Parasad, Appl. Phys. Lett. 68, 3549 (1996).

${ }^{15}$ G. Jordan, T. Kobayashi, W. J. Blau, S. Pfeiffer, and H.-H. Horhold, Adv. Funct. Mater. 13, 751 (2003). 